\title{
FG-4592 Improves Depressive-Like Behaviors through HIF-1-Mediated Neurogenesis and Synapse Plasticity in Rats
}

\author{
Gaifen $\mathrm{Li}^{1,2} \cdot$ Ming Zhao $^{1} \cdot$ Xiang Cheng ${ }^{1} \cdot$ Tong Zhao $^{1} \cdot$ Zhenlong Feng $^{1} \cdot$ Yongqi Zhao $^{1} \cdot$ Ming Fan $^{1,2,3}$. \\ Lingling Zhu ${ }^{1,2}$ (D)
}

Published online: 9 December 2019

(C) The American Society for Experimental NeuroTherapeutics, Inc. 2019

\begin{abstract}
Depression, plus the accompanying memory impairment, is one of the leading causes of disability worldwide. Thus, there is a critical need to develop new drugs based on distinct strategies. FG-4592, an inhibitor of prolyl hydroxylase, activates the hypoxia-inducible factor-1 (HIF-1) pathway, to produce multiple effects on cell properties. Here, we examined whether FG4592 has antidepressant effects, using a chronic unpredictable mild stress (CUMS) procedure to establish rodent depression models. We found that FG-4592 not only reversed depressive behaviors but also improved CUMS-induced memory impairment. Mechanistically, FG-4592 could play an important role in promoting hippocampal neurogenesis and synaptic plasticity. At the molecular level, FG-4592 was found to activate HIF-1 and cAMP-responsive element-binding protein/brain-derived neurotrophic factor signaling pathways in vivo, as well as promote the expression of postsynaptic density (PSD) proteins, PSD95 and Homer1. An examination of primary hippocampal neurons showed that FG-4592 promoted dendritic growth. Taken together, our results not only provide an experimental basis for the future application of FG-4592 in clinical treatment of depression but also support the argument that the HIF-1 signaling pathway is a promising target for the treatment of depression.
\end{abstract}

Key Words FG-4592 $\cdot$ CUMS $\cdot$ Neurogenesis $\cdot$ Synapse plasticity $\cdot$ HIF-1

\section{Introduction}

Depression, a severe psychiatric disorder, represents a serious and highly prevalent public health concern with huge impacts on the quality of life of affected individuals [1]. Depression is hypothesized to be a neuropsychiatric disease derived from neuronal dysfunction, as demonstrated by numerous studies reporting links between depression and dysfunction or structural changes within specific brain regions, for example, the

Gaifen Li and Ming Zhao contributed equally to this work.

Ming Fan

fanmingchina@126.com

$\triangle$ Lingling Zhu

linglingzhu@hotmail.com

1 Institute of Military Cognition and Brain Sciences, Academy of Military Medical Sciences, Beijing 100850, China

2 Beijing Institute for Brain Disorders, Capital Medical University, Beijing 100069, China

3 Co-Innovation Center of Neuroregeneration, Nantong University, Nantong 226001, China hippocampus $[2,3]$. Currently, there is no effective drug to treat depression, which exhibits a disturbingly complex pathogenesis $[4,5]$. Despite considerable progress made towards understanding the pathogenesis of depression, most antidepressants currently used in clinical practice have low efficacy and likely causes serious side effects [6-8]. Therefore, there is an urgent need to develop novel antidepressant strategies and new effective drugs for the treatment of depression.

Recent evidence indicates that exposure to intermittent hypoxia $(\mathrm{IH})$ has beneficial effects on neurological disorders, such as depression [9]. Specifically, IH can produce antidepressant-like effects by promoting neurogenesis in the adult hippocampus via brain-derived neurotrophic factor (BDNF) signaling pathway activation [10]. Using the same chronic unpredictable mild stress (CUMS) rat model employed in this study, Kushwah et al. validated the neuroprotective role of $\mathrm{IH}$ on depression, and identified an important role for the BDNF pathway [11]. In addition, hypoxiainducible factor-1 (HIF-1), which is the major transcription factor responding to hypoxia, may produce beneficial effects on depression, as its target genes erythropoietin (EPO) and vascular endothelial growth factor (VEGF) have been shown to elicit antidepressant effects in animal models. Indeed, 
VEGF signaling plays an essential role in the antidepressant action of the described drugs, and peripheral administration of EPO was sufficient to produce a robust antidepressant-like effect $[12,13]$. Collectively, these results suggest that activation of HIF-1 signaling is a promising strategy to improve depressive behaviors.

HIF- 1 is a heterodimer consisting of $\alpha$ and $\beta$ subunit, with HIF- $1 \alpha$ playing a regulatory role through its extreme sensitivity to changes in oxygen availability. Under normoxic conditions, HIF-1 $\alpha$ is hydroxylated by prolyl hydroxylases (PHDs) and then targeted to undergo ubiquitin-proteasomal degradation [14]. Therefore, blocking of PHD enzymatic activity likely results in activation of the HIF-1 pathway and subsequent promotion of target gene expression, thereby producing a dramatic impact on cell processing. PHD inhibitors act as hypoxia-mimicking agents, leading to a hypoxic environment for cells and tissues. As a result, it is strongly suggested that PHD inhibitors exhibit beneficial effects on depression.

FG-4592, also known as roxadustat, is an oral PHD inhibitor that is used for the treatment of anemia in patients with chronic kidney disease (CKD) [15]. In the present study, FG4592 was found to reverse depression-like behaviors in CUMS model rats and concurrently improve spatial memory impairment. Additionally, FG-4592 was shown to promote hippocampal neurogenesis and synaptic plasticity in vivo and in vitro. These results clearly demonstrated that FG4592 is a promising therapeutic agent for depression.

\section{Materials and Methods}

\section{Animals}

Two-month-old male Sprague-Dawley (SD) rats, weighing $\sim 250 \mathrm{~g}$, were obtained from Beijing Vital River Laboratory (Beijing, China). In addition, 2-month-old male Thy1-YFP transgenic mice (C57BL/6 background), weighing $\sim 20 \mathrm{~g}$, were included as described above [16]. All animals were housed in groups of four or five and maintained under standard laboratory conditions (12/12-h light/dark cycle, $22 \pm 2{ }^{\circ} \mathrm{C}$, ad libitum access to food and water). All animal experimental procedures fully complied with related laboratory animal regulations.

\section{Ethics Statement}

All experimental procedures were approved by the Institutional Animal Care and Use Committee of Institute of Cognition and Brain Sciences (No. IACUC2017026) and performed in accordance with the guidelines. During treatment, behavioral testing and tissue collection procedures were devised to minimize the potential pain and distress of the animals used in this study. All of the rats and mice were frequently monitored, at least three times a week, for health status.

\section{Drugs Administration}

FG-4592 and fluoxetine were ordered from Selleck Chemicals (Houston, TX). For FG-4592 administration, $200 \mathrm{mg}$ of FG-4592 was dissolved in $2.857 \mathrm{~mL}$ of dimethyl-sulfoxide (DMSO) as a stock solution, which was subsequently diluted with $0.9 \% \mathrm{NaCl}$ into working concentrations for intraperitoneal injections. The final concentration of DMSO was less than $5 \%(v / v)$. Fluoxetine was dissolved with $0.9 \% \mathrm{NaCl}$ directly to its working concentration $(10 \mathrm{mg} / \mathrm{kg})$, and intraperitoneally injected for 2 weeks. For the proliferation assay, BrdU (B5002, Sigma-Aldrich, St. Louis, MO) was dissolved to $10 \mathrm{mg} /$ $\mathrm{mL}$ in $0.9 \% \mathrm{NaCl}$ for intraperitoneal injection into animals at a dosage of $50 \mathrm{mg} / \mathrm{kg}$ body weight six times per day.

\section{CUMS Procedure}

Identical procedures were performed to establish the CUMS depression model in rats and mice. The following stressors were imposed for 5 weeks: food deprivation overnight, water deprivation overnight, cage tilt $\left(45^{\circ}\right)$ for $24 \mathrm{~h}$, overnight illumination, sawdust bedding for $24 \mathrm{~h}$, swimming at $4{ }^{\circ} \mathrm{C}$ for $5 \mathrm{~min}$, physical restraint for $2 \mathrm{~h}$, alterations of light/dark cycle, tail pinch for $1 \mathrm{~min}$, and noise stimulation for $2 \mathrm{~h}$. Stressors were randomly scheduled to produce an unexpected mild stress effect. Control animals were left uninterrupted, except for regular cage cleaning.

\section{Sucrose Preference Test}

Animals were first trained to consume $1 \%$ sucrose solution from two different bottles for $24 \mathrm{~h}$ before conducting the formal experiment. After deprivation for $22 \mathrm{~h}$, each animal was allowed free access to $1 \%$ sucrose and pure water from two different bottles. To avoid bottle side preference, the two bottles were switched after $1 \mathrm{~h}$. Amounts in the two bottles were measured after $2 \mathrm{~h}$ and sucrose preference was calculated according to the following formula: sucrose preferences $(\%)=$ sucrose water consumption / (sucrose + pure) water consumption $\times 100 \%$. Observers were blinded to the treatment and group of tested animals.

\section{Open-Field Test}

Open-field test was performed in a quiet environment. Each animal was placed in the center of the bottom of a box (open field $60 \mathrm{~cm}$ length, $60 \mathrm{~cm}$ width, and $45 \mathrm{~cm}$ height). To ensure that the test results were free from any previous residual effect, the inner wall and bottom of the box were thoroughly cleaned 
before each test. The activity of each animal was monitored for 5 min using an ANY-maze behavior analysis system (Stoelting, Wood Dale, IL).

\section{Tail Suspension Test}

Animals were individually suspended by their tails from a bar for $5 \mathrm{~min}$. Immobility duration during the 5 -min suspension period was recorded. Observers were blinded to the treatment and group of tested animals.

\section{Forced Swimming Test}

Animals were individually placed in a transparent glass container $40 \mathrm{~cm}$ in depth, in which they were forced to swim, because the bottom of the pool was beyond their reach. Total immobility time was recorded during the 5min testing period. Time when the animal performed only slight movements necessary to keep its head above water was considered immobile time.

\section{Morris Water Maze}

The water maze used in this study comprised a circular tank $160 \mathrm{~cm}$ in diameter with a platform, filled with tap water at a temperature of $23 \pm 2{ }^{\circ} \mathrm{C}$. Different shapes and colors were posted along the curtains of the tank, which served as spatial reference cues. A camera was mounted above the tank to record swimming tracks in the water maze. During acquisition trials lasting 5 days, the platform was submerged $2 \mathrm{~cm}$ below the water surface, and rats were placed into the maze at one of four quadrants facing the curtain of the tank. Rats were allowed $90 \mathrm{~s}$ to search the platform. If rats failed to find the platform, they were guided to and kept on the platform for $10 \mathrm{~s}$. Four trials per day were conducted with a minimum intermission of $1 \mathrm{~h}$ between trials. Escape latency, which is indicative of spatial memory acquisition, was recorded for each trial. On day 6 , the platform was removed, a probe test was conducted, and rats were put into the water at one quadrant (usually the farthest quadrant from the hidden platform). Each rat was allowed to swim in the water for $90 \mathrm{~s}$; during which, the first time the rat found the platform location and number of platform crossings was recorded.

\section{Novel Object Recognition}

The experimental device was $60 \mathrm{~cm}$ in length, $60 \mathrm{~cm}$ in width, and $45 \mathrm{~cm}$ in height. The experiment was divided into three stages: adaptation, familiarity, and testing. Identification of objects $\mathrm{A}$ and $\mathrm{B}$ was performed for two different materials and colors. In the adaptive phase, there was no object in the recognition apparatus, and rats were given $10 \mathrm{~min}$ to adapt to the environment. In the training phase, there were two identical objects $\mathrm{A}$ in the recognition apparatus, and rats were placed in a familiar location in the environment for $10 \mathrm{~min}$. In the testing phase, one object A was replaced with object $B$ in the recognition apparatus, and the video tracing system recorded the time rats spent identifying object B. To evaluate novel object recognition, recognition index (RI) was calculated using the formula $\mathrm{RI}=N-F /(N+F)$, in which $N$ represents the total time the rat's nose touched object $\mathrm{B}$, and $F$ represents the total time of the rat's nose touched object A.

\section{Immunofluorescence}

Animals were deeply anesthetized with intraperitoneal injection of $2 \%$ sodium pentobarbital, followed by perfusion with $100 \mathrm{~mL}$ of heparinzed $0.9 \%$ saline and $200 \mathrm{~mL} 4 \%$ paraformaldehyde (PFA). Brains were postfixed at $4{ }^{\circ} \mathrm{C}$ for $4-6 \mathrm{~h}$ in $4 \%$ PFA, followed by $15 \%$ sucrose for $24 \mathrm{~h}$, and subsequent dehydration in $30 \%$ sucrose at $4{ }^{\circ} \mathrm{C}$ for $48 \mathrm{~h}$. After sucrose saturation, brains were cut in the coronal plane into $40-\mu \mathrm{m}$ thick sections with a freezing microtome (model: E, Thermo, Waltham, MA) and processed for immunofluorescence.

Immunostaining was performed in accordance with a procedure routinely performed in our laboratory. A mouse monoclonal anti-BrdU antibody (1:1000; Sigma-Aldrich), and Alexa Fluor 488 goat anti-mouse secondary antibodies (1:500; Invitrogen, Carlsbad, CA) were used in this study.

\section{Western Blotting}

Hippocampus and cultured hippocampal neurons were homogenized in lysis buffer (RIPA plus protease inhibitor cocktail) on ice for $30 \mathrm{~min}$, and subsequently centrifuged at $12000 \times g$ for $10 \mathrm{~min}$ at $4{ }^{\circ} \mathrm{C}$. The resulting supernatants were collected as the whole cell lysates. Protein quantification was determined by bicinchoninic acid assay. A total $50 \mu \mathrm{g}$ of protein was resolved by sodium dodecyl sulfate-polyacrylamide gel electrophoresis and subsequently immunoblotted onto a polyvinylidene difluoride membrane, which was then blocked with 5\% nonfat milk at room temperature for $1 \mathrm{~h}$. After washing three times ( $5 \mathrm{~min}$ each) in Tris-buffered saline containing Tween 20, the membrane was incubated with the indicated primary antibodies at $4{ }^{\circ} \mathrm{C}$ with shaking overnight. TPrimary antibodies were as follows: HIF-1 $\alpha$ (1:2000, Novus Biologicals, Centennial, CO), EPO (1:2000, Abcam, Cambridge, UK), VEGF (1:2000, Abcam, Cambridge, UK), PSD95 (1:5000, Cell Signaling Technology (CST), Danvers, MA), Homer1 (1:2000, CST), BDNF (1:5000, CST), pCREB (1:2000, CST), and CREB (1:2000, CST). In addition, a control mouse monoclonal $\beta$-actin primary antibody was obtained from Sigma-Aldrich (1:10000, A5316). Subsequently, the membrane was washed, as described above, and incubated with secondary antibodies at room temperature for $2 \mathrm{~h}$. The membrane was then developed with an enhanced 
chemiluminescence detection system (Applygen, Beijing). Quantification of protein band intensities was normalized to $\beta$-actin. Statistical analysis was performed with PhotoShop (Adobe, San Jose, CA) and Prism 6.0 software (GraphPad, La Jolla, CA).

\section{Quantitative RT-PCR}

Total RNA was extracted from the hippocampus of rat brains using Trizol reagent (Invitrogen). First-stand cDNA of each sample was synthesized using an MLV reverse transcription kit (TAKARA, Kusatsu, Japan) according to the manufacturer's instructions. cDNA was used as a template for quantitative real-time PCR using SYBR Green Master Mix (Applied Biosystems, Foster City, CA). Gene expression was calculated relative to $\beta$-actin. Samples were assayed in triplicate. Primer sequences were as follows: EPO 5'-CTTGCTACAGATTC CTCTG-3' and 5 '-TGTTCTTCCACCTTCATTC-3'; VEGF 5 '-GCAGTGCTCCCCATCCGCTG-3' and 5 '-TGCTCGTC CGACAGCTGGGA-3'; $\beta$-actin 5 '-GGCTGTATTCCCCT CCATCG-3' and 5 '-CCAGTTGGTAACAATGCCATGT-3'.

\section{Cell Culture and Treatments}

Primary culture of hippocampal neurons was prepared from $24 \mathrm{~h}$ newborn SD rats. Hippocampi were dissected, incubated for $30 \mathrm{~min}$ in trypsin/EDTA at $37^{\circ} \mathrm{C}$, and, subsequently, mechanically dissociated. Cells were plated at a density of $9 \times$ $10^{4}$ cells $/ \mathrm{cm}^{2}$ on poly-L-lysine-coated $24-$ well plates and maintained in Neurobasal medium supplemented with $1 \%$ B27 supplement and $1 \%$ penicillin/streptomycin. Cells were incubated for 8 days at $37^{\circ} \mathrm{C}$ and $5 \% \mathrm{CO}_{2}$ and $99 \%$ humidity. Hippocampal primary neurons were treated with $50 \mu \mathrm{M}$ and $10 \mu \mathrm{M}$ FG-4592 for $72 \mathrm{~h}$, starting from the second day in vitro. Microtubule-associated protein 2 (MAP2) staining and Western blotting were performed after cell collection.

\section{Statistical Analysis}

All data are displayed as mean \pm standard error of the mean (SEM). Statistical testing was conducted using GraphPad Prism 6.0 software. One-way ANOVA with Tukey's multiple comparisons tests was applied to analyze and compare continuous variables of sucrose preference test (SPT), open-field test (OFT), tail suspension test (TST), forced swimming test (FST), Morris water maze (MWM), novel object recognition (NOR), and Western blotting optical density calculations. Data for body weight change was analyzed by two-way ANOVA of repeated measurements with Tukey's post hoc tests. A $p<0.05$ (two-tailed) was considered statistically significant for all tests.

\section{Results}

\section{Effects of Different Doses of FG-4592 on Rat Body Weight}

To investigate the potential effects of FG-4592 on CUMSinduced depression, different dosages of FG-4592 were administered to CUMS rats. According to previous reports [17-19], three dosages of FG-4592 $(5,10,20 \mathrm{mg} / \mathrm{kg} /$ day $)$ were selected. As shown in Fig. 1A, rats were subjected to the CUMS procedure for 3 weeks, and thereafter intraperitoneally administered FG-4592 at a dosage of 5, 10, or $20 \mathrm{mg} / \mathrm{kg} / \mathrm{day}$ or fluoxetine (as a positive control) for 2 weeks. We first evaluated the changes in the body weight of rats in different groups. Compared with the control group rats, CUMS group rats experienced a perceptibly slow increase in body weight. Administration of FG-4592 or fluoxetine did not have an evident impact on the body weight of CUMS rats. However, a high dosage of FG-4592 (20 mg/kg/day) was found to have a significant impact on body weight gain (Fig. 1B).

\section{Different Dosages of FG-4592 Reversed CUMS-Induced Depression-like Behaviors}

To confirm the success of CUMS depression model replication and the effect of drug injection on depressive behaviors in model animals, rats were evaluated by SPT, TST, FST, and OFT behavioral tests. As shown in Fig. 2A, the sucrose preference of CUMS rats was significantly reduced compared with that of the control group. However, this trend was reversed by FG-4592 or fluoxetine treatment, which exhibited no significant dose-related differences. In TST, CUMS rats were immobile for a longer time than control group rats, but this effect was reversed by FG-4592 or fluoxetine treatment. FG-4592 at $10 \mathrm{mg} / \mathrm{kg}$ produced the best effect (Fig. 2B). In FST, the trend was similar to the results of TST, in that CUMS rats were immobile for a longer period of time than controls, and this was reversed by FG-4592 or fluoxetine treatment; no obvious dose-dependent effect was detected for FG4592 (Fig. 2C). In OFT, CUMS rats exhibited significantly decreased spontaneous activity compared with the control group rats, and showed recovery with FG-4592 or fluoxetine treatment. We noticed that medium-dose FG4592 produced a more stable effect, consistent with the antidepressant effect of fluoxetine (Fig. 2D-F). These results indicated that the CUMS depression model could be successfully replicated, which means that FG-4592 could effectively reverse CUMS-induced depression-like behaviors. Balancing the negative effect of high dose FG-4592 $(20 \mathrm{mg} / \mathrm{kg} /$ day) on body weight with the stable improvement observed with a medium dose $(10 \mathrm{mg} / \mathrm{kg} / \mathrm{day})$ on 
Fig. 1 Experimental design and effects of c on body weight. (A) Schematic representation of the experimental design. Animals were adapted for 3 days before the onset of chronic unpredictable mild stress (CUMS) stress.

Subsequently, animals were subjected to a 5-weeks CUMS regimen followed by behavioral tests. SPT: Sucrose preference test, TST: Tail suspension test, FST: Forced swimming test, OFT: Open-field test, MWM: Morris water maze, NOR: Novel object recognition. (B) Effects of FG4592 on body weight. Animal subjected to CUMS exhibited reduced body weight compared with the control group, whereas FG-4592 had no effect on body weight. $\mathrm{CON}=$ control group; CUMS = CUMS group; FG-5/10/ $20=$ FG-4592 $(5,10$, and $20 \mathrm{mg} / \mathrm{kg}$ ) groups; $\mathrm{C}+\mathrm{FG}=$ CUMS+FG-4592 $(5,10$, and $20 \mathrm{mg} / \mathrm{kg}$ ) groups. All data are expressed as mean \pm SEM, $n=6$, $* p<0.05, * * p<0.01$ versus control (CON) group

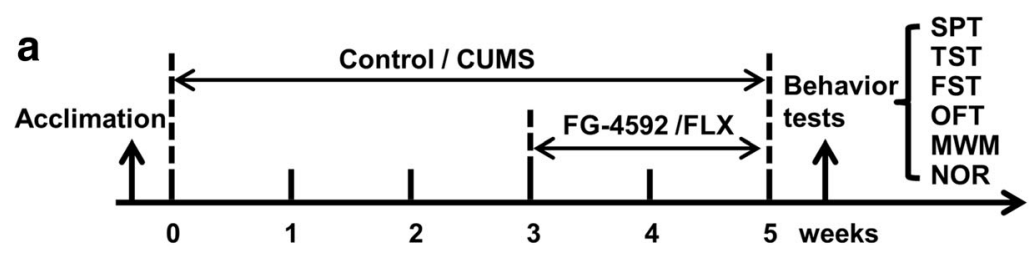

b

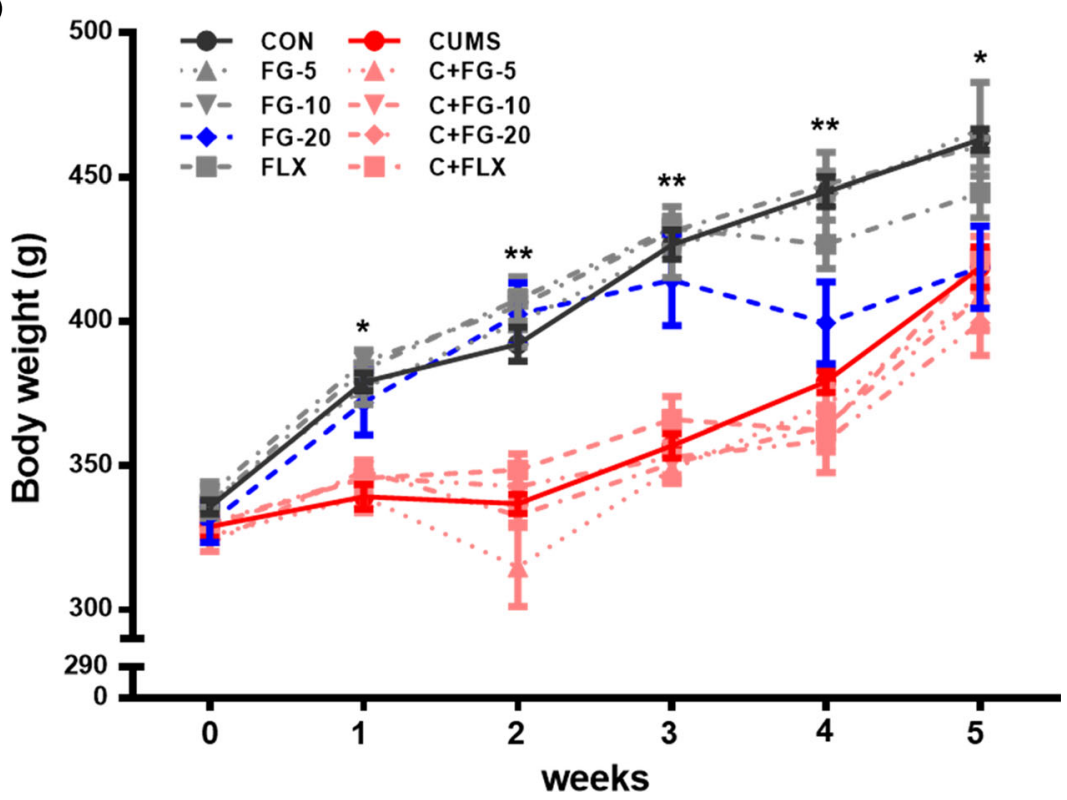

depression-like behaviors, we decided to use a dose of $10 \mathrm{mg} / \mathrm{kg} /$ day for the following studies.

\section{FG-4592 Improved CUMS-Induced Impairments of Learning and Memory}

Apart from the well-defined depressive symptoms, depression is conventionally complicated by cognitive deficits, such as impairments in learning and spatial memory [20, 21]. In support of this argument, we examined changes in learning and spatial memory by MWM and NOR tests. As shown in Fig. 3A, during the 5 days of acquisition training, average escape latency of CUMS rats was increased compared with that of the control rats. However, FG-4592 or fluoxetine treatment was found to significantly reduce the time of escape latency under CUMS conditions. This result suggests that FG-4592 effectively improved CUMSinduced learning dysfunction. In the probe test, CUMS resulted in obvious spatial memory impairments, as demonstrated by fewer successful crossings and shorter latency compared with the control group. However, all of these parameters were reversed by FG-4592 or fluoxetine treatment (Fig. 3B, C). These results reinforce the conclusion that FG-4592 elicits an evident beneficial effect on CUMSinduced memory impairment. Similarly, NOR results showed that CUMS group rats had significantly shorter novel object exploration times compared with the control group; this discrepancy was nevertheless reversed by FG4592 or fluoxetine treatment (Fig. 3D). Collectively, these results demonstrated that FG-4592 treatment produced a favorable effect on CUMS-induced learning and memory impairment in rats.

\section{FG-4592 Rescued Decreased of Hippocampal Neurogenesis and Synaptic Deficits Induced by CUMS}

Previous studies found that depression is associated with decreased neurogenesis [22]. To evaluate this effect, BrdU was administered at the experimental endpoint to determine the influence of FG-4592 on survival of newborn neurons in the hippocampus. As shown in Fig. 4A and $\mathrm{B}$, a modulate number of BrdU-positive cells were observed in the control group. In contrast, a significant decrease in the number of BrdU-positive cells was observed in CUMS rats. However, CUMS rats treated with FG4592 displayed a significant increase in the number of 
Fig. 2 Effects of different doses of FG-4592 on depression-like behaviors in rats subjected to chronic unpredictable mild stress (CUMS). A-F, Effect of FG-4592 on depression-like behaviors in CUMS rats. (A) Sucrose preference test, (B) Tail suspension test, (C) Forced swimming test. (D) Entries into the central area were recorded in the open field test. (E) Total distance in the central area was recorded in the open field test, $(\mathrm{F})$ Time spent in the central area was recorded in the open field test
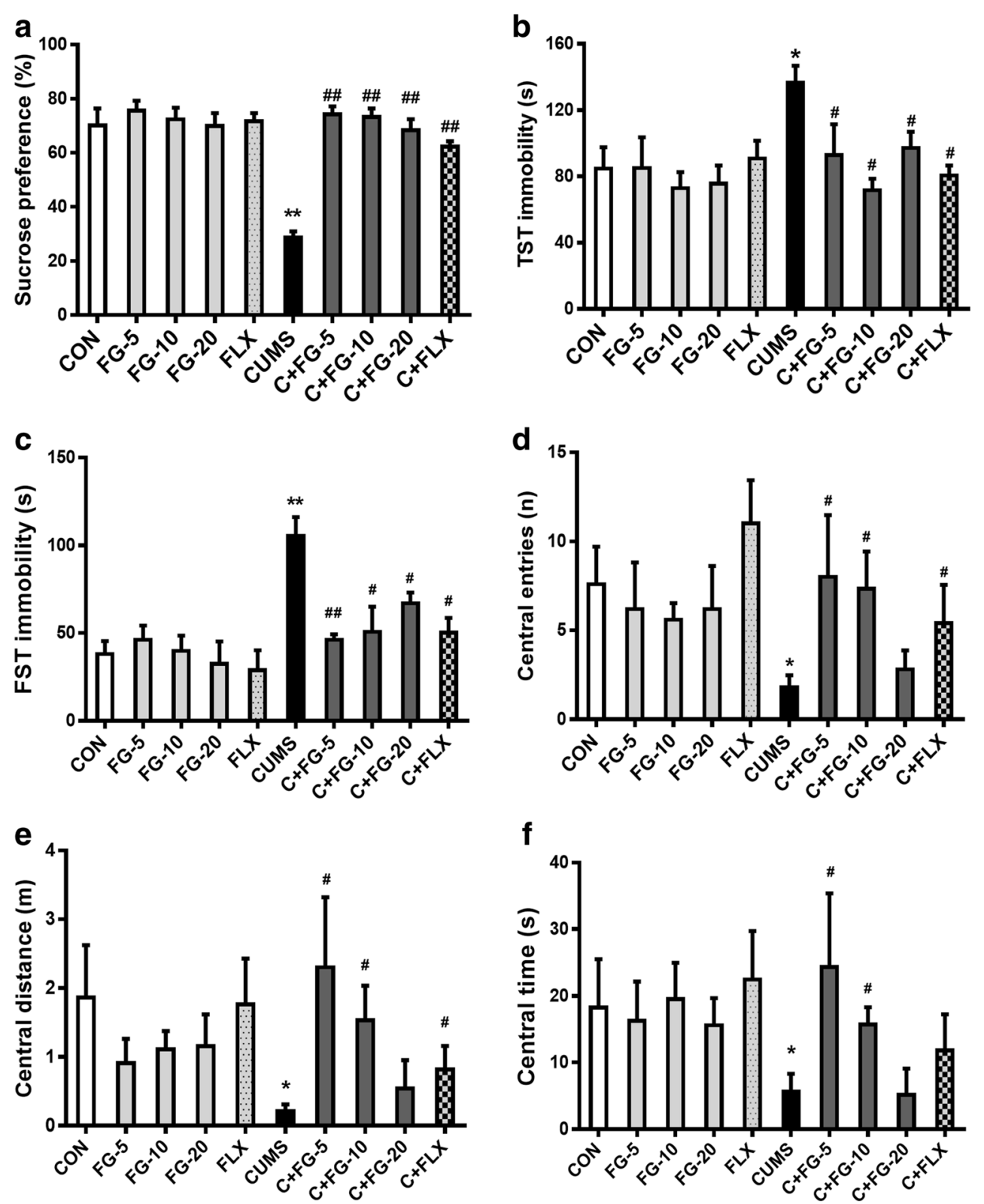

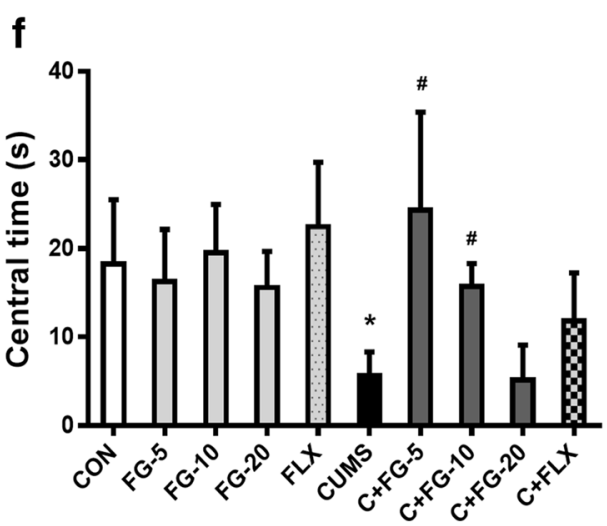

BrdU-positive cells, indicating that FG-4592 alleviated the negative effect of CUMS on neurogenesis.

As FG-4592 is believed to improve the memory impairment caused by CUMS, we examined its effect on synaptic plasticity, an important neurochemical foundation of memory [23]. Thy1YFP transgenic mice, which express the fluorescent yellow fluorescent protein (YFP) in neurons, were subjected to the CUMS paradigm as performed in rats, including treatment with or without $10 \mathrm{mg} / \mathrm{kg} /$ day FG-4592. The results of brain slice examination showed that compared with the control rats, a lower density of dendritic spines was present in hippocampal CA1 pyramidal cells of the CUMS group rats. Interestingly, FG-4592 treatment was found to obviously increase the density of dendritic spines under CUMS conditions (Fig. 4C, D), indicating that FG-4592 promoted synaptic plasticity.

\section{FG-4592 Unregulated HIF-1a Signaling and Other Proteins in CUMS Rat Hippocampus}

To explore potential molecular mechanisms underlying the beneficial effect of FG-4592, we first examined changes of HIF-1 signaling in vivo. As shown in Fig. $5 \mathrm{~A}$ and $\mathrm{B}, \mathrm{FG}-$ 4592 not only increased protein levels of HIF-1 $\alpha$, VEGF, and EPO in the hippocampus of CUMS rats, compared with the control group, but also rescued decreased protein levels of HIF- $1 \alpha$, VEGF, and EPO in CUMS model animals. $q$ PCR results showed that FG-4592 increased mRNA levels of EPO and VEGF, and concurrently, reversed the reduction of EPO and VEGF mRNA levels in the CUMS group (Fig. 5C, D). These results provided fresh evidence of HIF-1 pathway activation in the FG-4592-treated group. 
Fig. 3 Chronic unpredictable mild stress (CUMS)-induced memory impairment was reversed by FG-4592. (A) Escape latency to find the hidden platform across four trials during the acquisition training period of ten rats in each group. (B, C) The mean number of platform crossing and mean latency in first-time passing the location of the original platform, respectively, during the probe test. (D) Discrimination index of novel object recognition was evaluated. All data are expressed as mean $\pm \mathrm{SD}, n=10, * p<0.05$,

$* * p<0.01$ versus control (CON) group; ${ }^{\#} p<0.05,{ }^{\# \#} p<0.01$ versus CUMS group
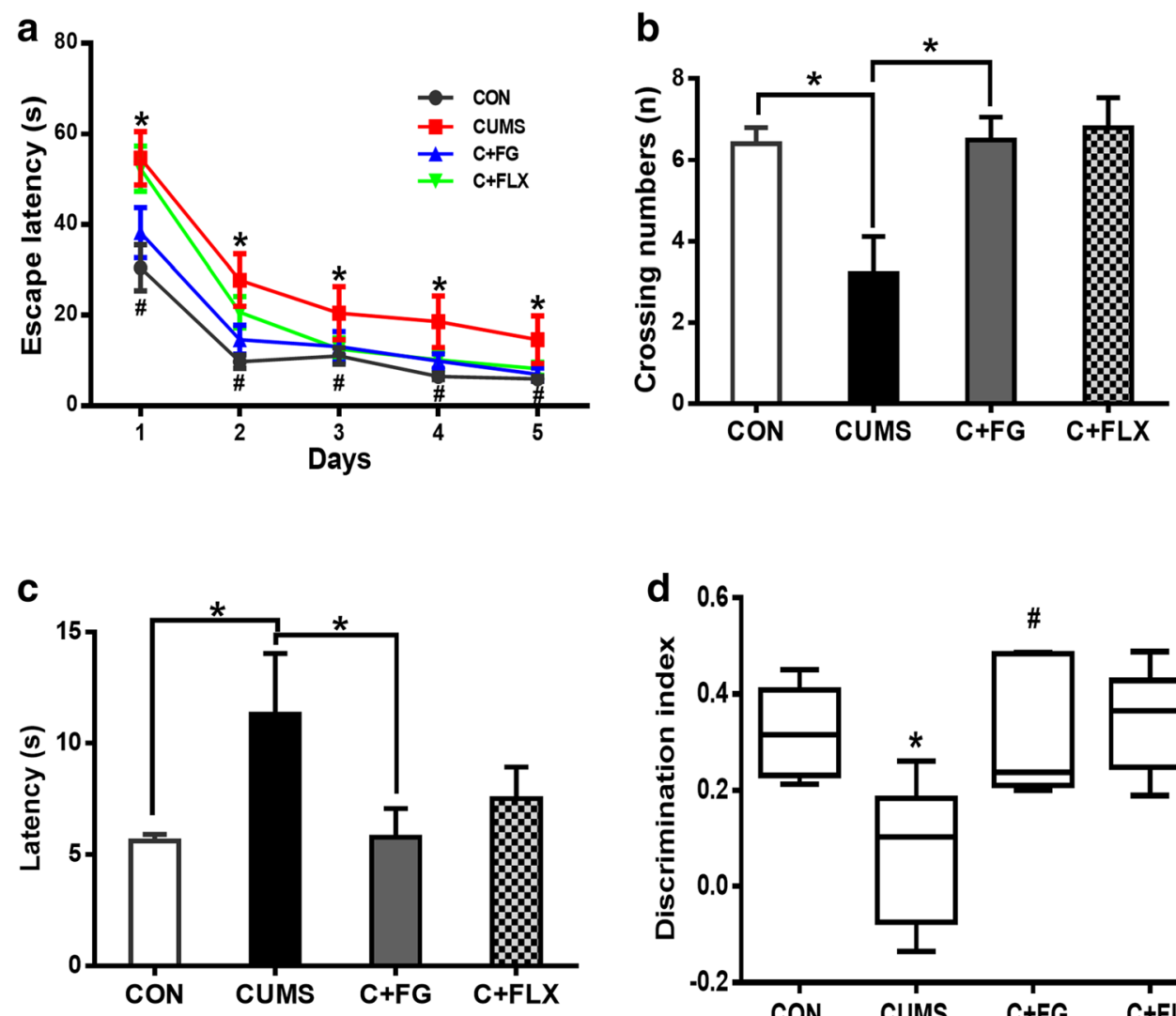

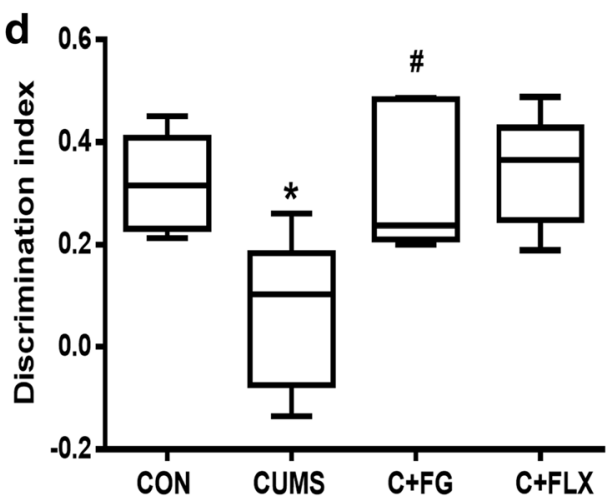

In addition, we detected changes in memory-related CREB/BDNF signaling in the hippocampus of CUMS rats [24]. Western blot results showed that protein levels of pCREB and BDNF (to a lesser extent) were decreased in the CUMS group. However, this decrease was rescued in the FG4592+CUMS group (Fig. 5E, F), indicating that CREB/ BDNF signaling was likely involved in the beneficial effect of FG-4592 on memory impairment. Interestingly, CUMS caused reduced protein levels of PSD95 and Homer1, two important synaptic density proteins, but this was also reversed by FG-4592 treatment (Fig. 5G, H), indicating that PSD95 and Homer1 could be target proteins for the effect of FG4592 on synaptic plasticity.

\section{FG-4592 Promoted Synaptic Growth in Primary Hippocampal Neurons In vitro}

IH can promote synaptic growth in vivo and in vitro [25]. Thus, we speculated that FG-4592 could promote synaptic growth by activating the HIF-1 pathway. We isolated and cultured hippocampal neurons from newborn rats (within $24 \mathrm{~h}$ ) to ascertain whether FG-4592 promoted dendritic growth in vitro. As shown in Fig. 6A-C, after FG-4592 treatment, the dendritic length of hippocampal neurons was noticeably increased compared with control hippocampal neurons. However, no effect of FG-4592 on neuron number was detected. In addition, FG-4592 dose- dependently increased protein levels of HIF-1, and its target genes EPO and VEGF, as well as BDNF and PSD95 (Fig. 6D-G). These results arguably offer proof that FG4592 promotes synaptic growth both in vivo and in vitro by up-regulating HIF- $1 \alpha$ and its target genes.

\section{Discussion}

In this study, we aimed to determine if the PHD inhibitor FG-4592 exhibits antidepressant properties in a CUMS rat model. The results of the behavioral tests showed that FG4592 not only reversed depression-like behaviors but also improved the memory impairment caused by CUMS. In addition, FG-4592 was found to promote neurogenesis and synaptic plasticity under CUMS conditions. At the molecular level, FG-4592 activated the HIF-1 signaling pathway, and increased expression of postsynaptic structural proteins PSD95 and Homer1 (Fig. 7). Apart from the above findings, supporting evidence was found concerning the instrumental role of FG-4592 in dendritic growth through the HIF-1 pathway. We believe these findings collectively demonstrate that FG-4592 is a promising and effective agent for treatment of depression.

Building on findings from previous reports, we conducted an investigation to establish if the HIF-1 pathway is a potential target for treatment of depression. Previous studies found that 

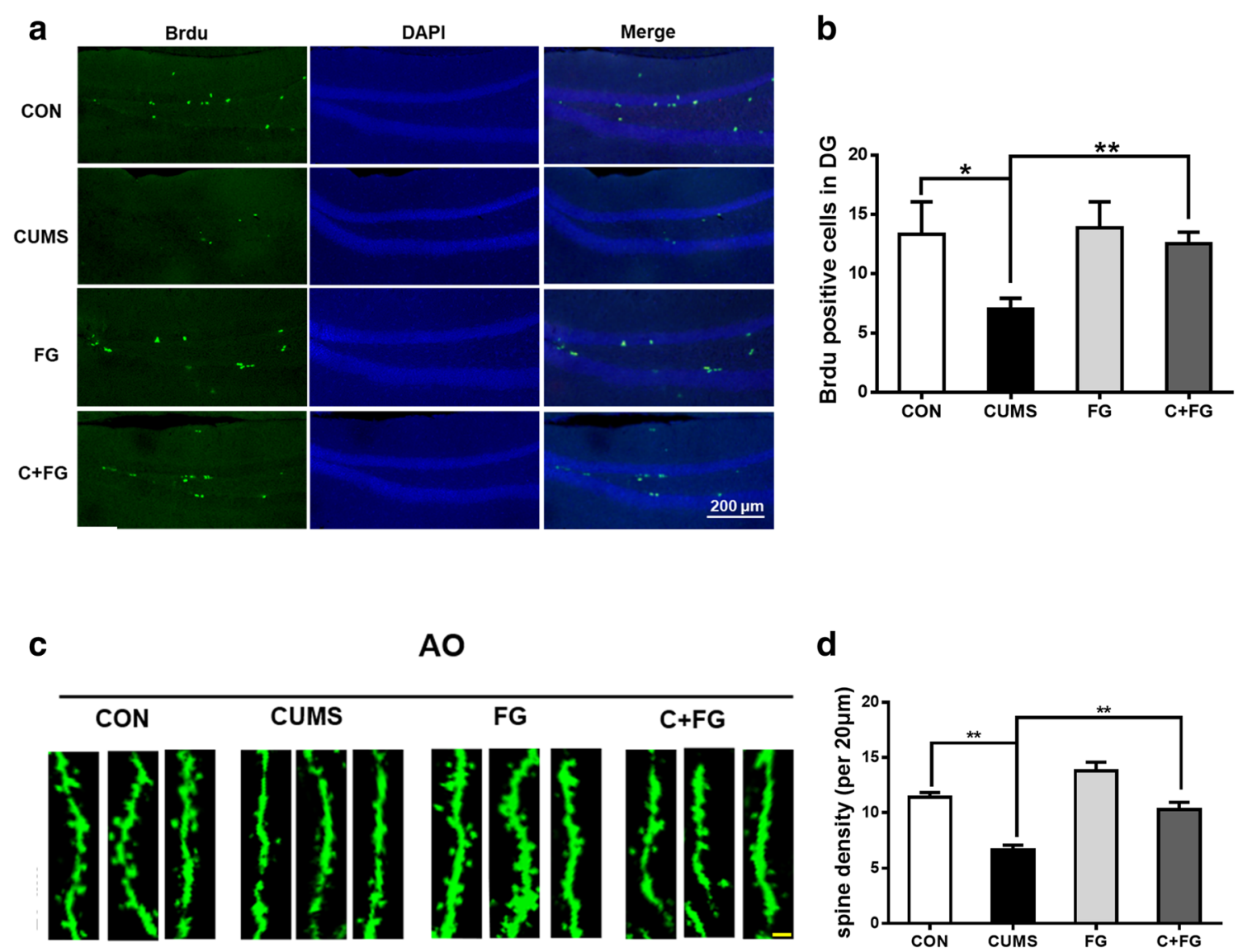

Fig. 4 FG-4592 promoted hippocampal neurogenesis and synaptic plasticity in mice subjected to chronic unpredictable mild stress. (A) Representative image of BrdU-positive (newborn) cells in the subgranular zone-granule cell layer of the hippocampus in mice. (B) Histograms showing the number of BrdU-positive cells as shown in (A). Data are expressed as mean \pm SEM, $n=3, * p<0.05, * * p<0.01$.

(C) Representative dendritic segments of CA1 pyramidal neurons (AO $=$ apical oblique dendrite) of Thyl-YFP mice, scale bar $=1 \mu \mathrm{m}$. (D) Dendritic spine density expressed as the number of spines normalized to $20 \mu \mathrm{m}$ of dendritic length; data are expressed as mean \pm SEM, $n=3$, $* p<0.05, * * p<0.01$

IH has neuroprotective role in CUMS rats, as markedly increased HIF-1 pathway activation resulted in sustained expression of its target gene EPO and the neurotrophic factor BDNF $[10,11]$. Moreover, it was previously reported that peripheral administration of EPO, a HIF-1 master target gene, is sufficient to produce robust antidepressant-like effects as evidenced by behavioral tests, and induces expression of neurotrophic genes such as BDNF [12]. Recently, Deyama et al. found that the antidepressant-like and neurotrophic actions of BDNF require VEGF signaling, and there is a reciprocal interaction between these two molecules [26]. These findings suggest that HIF-1 pathway activation and increased BDNF have important roles in mediating mild hypoxia-induced antidepressant effects. Given that FG-4592, an oral PHD inhibitor, has already been approved for treatment of anemia with CKD (including validation of its tolerance and safety), FG-4592 has a good chance to also be a practicable therapeutic option for the treatment of depressive disorders.

It is well established that neurogenesis persists in specific regions of the adult brain in mammals [27]. Chronic exposure

or severe forms of stress (as mimicked in the CUMS model) can induce long-lasting reductions in neurogenesis, in particular, within the hippocampus [28]. Indeed, hippocampal neurogenesis is required for the behavioral effects of antidepressants [29]. Hence, stimulation of neurogenesis is regarded as a promising strategy for identifying new antidepressant targets. Consistent with previous reports [30], CUMS resulted in reduced neurogenesis in our study, as evidenced by decreased of BrdU staining in the hippocampus. However, this reduction was reversed by FG-4592, suggesting that FG-4592 plays an important role in neurogenesis. Given that VEGF can stimulate neurogenesis in vitro and in vivo [31], and chronic mild hypoxia can promote hippocampal neurogenesis through the Notch1 pathway [32], we speculated that the induction of neurogenesis by FG-4592 is probably attributable to its activating role in the HIF-1 pathway.

In addition to decreasing BDNF expression, which plays critical roles in both neurogenesis and synaptic plasticity, FG4592 exerted a similar influence on expression of PSD95 and Homer1, two synaptic structural proteins [33, 34], indicating 


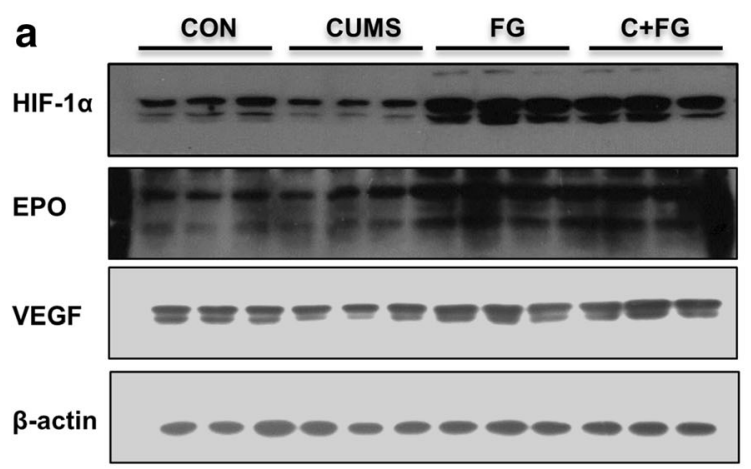

b

C

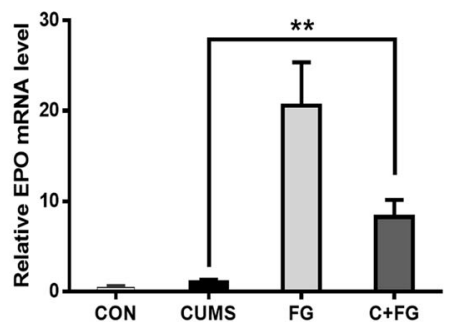

d
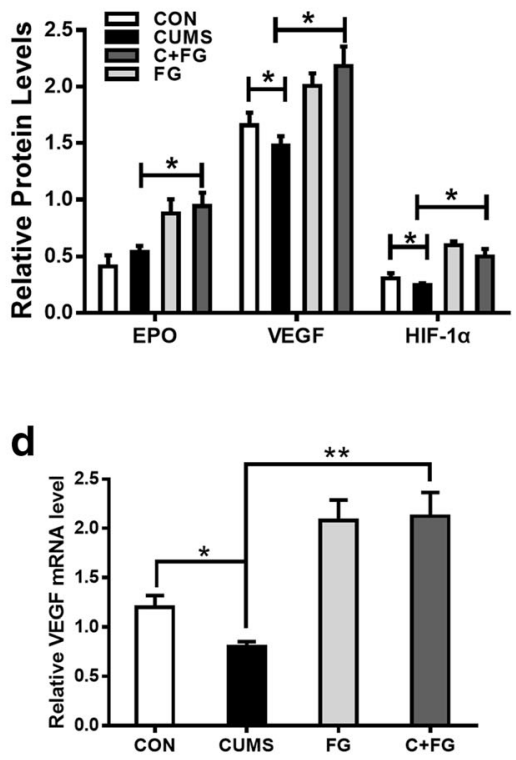

e

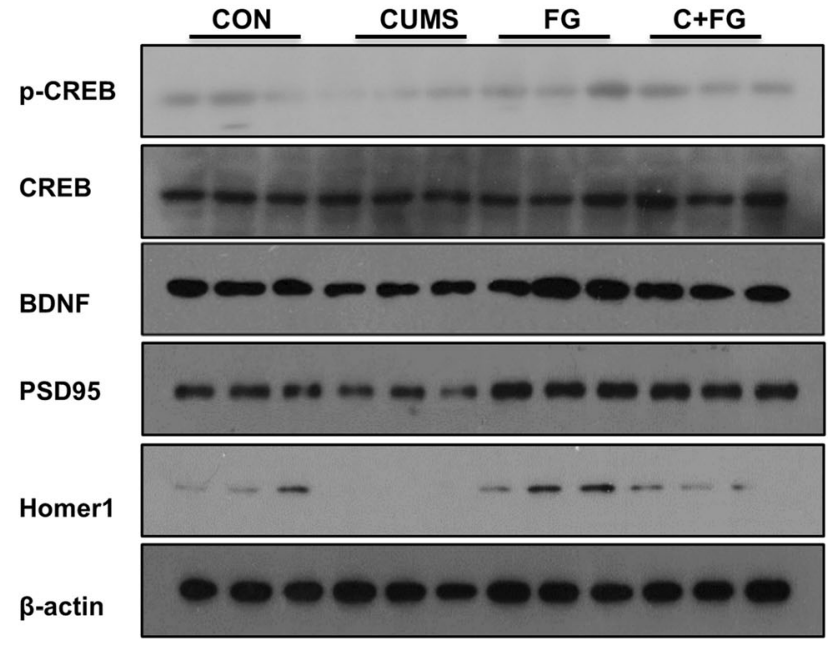

f

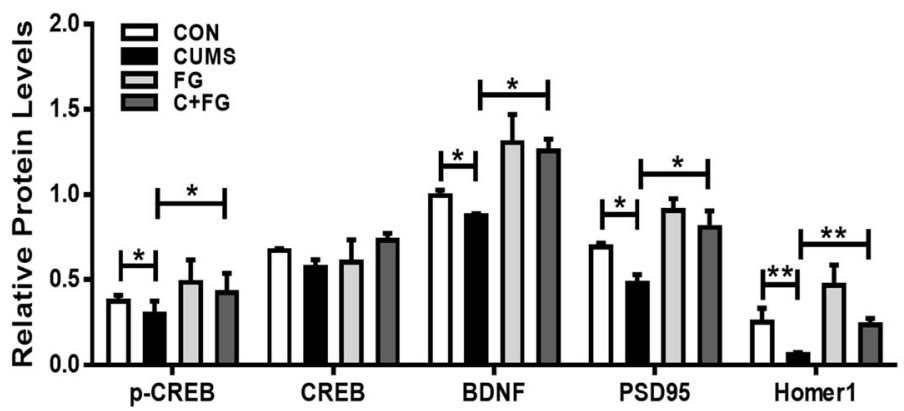

Fig. 5 FG-4592 activated hypoxia-inducible factor (HIF-1) and cAMP response element-binding protein (CREB)/brain-derived neurotrophic factor (BDNF) pathways, and increased expression of postsynaptic density proteins. (A, B) Western blots showing expression of HIF-1 $\alpha$ and downstream target genes vascular endothelial growth factor (VEGF) and erythropoietin (EPO) in the hippocampus. Histograms on the right show relative protein band density. (C, D) Real-time $q$ PCR was used to assess mRNA levels of VEGF and EPO in the hippocampus. (E, F) Western blots of cAMP-response element-binding protein (CREB), phosphorylate CREB (p-CREB), and brain-derived neurotrophic factor (BDNF) in the hippocampus. Histograms on the right show relative protein band density. Western blots of postsynaptic density protein 95 (PSD95) and Homer1 in the hippocampus were shown. The right histograms showed the relative protein band density. All data are expressed as mean \pm SEM, $n=3, * p<0.05, * * p<0.01$ 
a

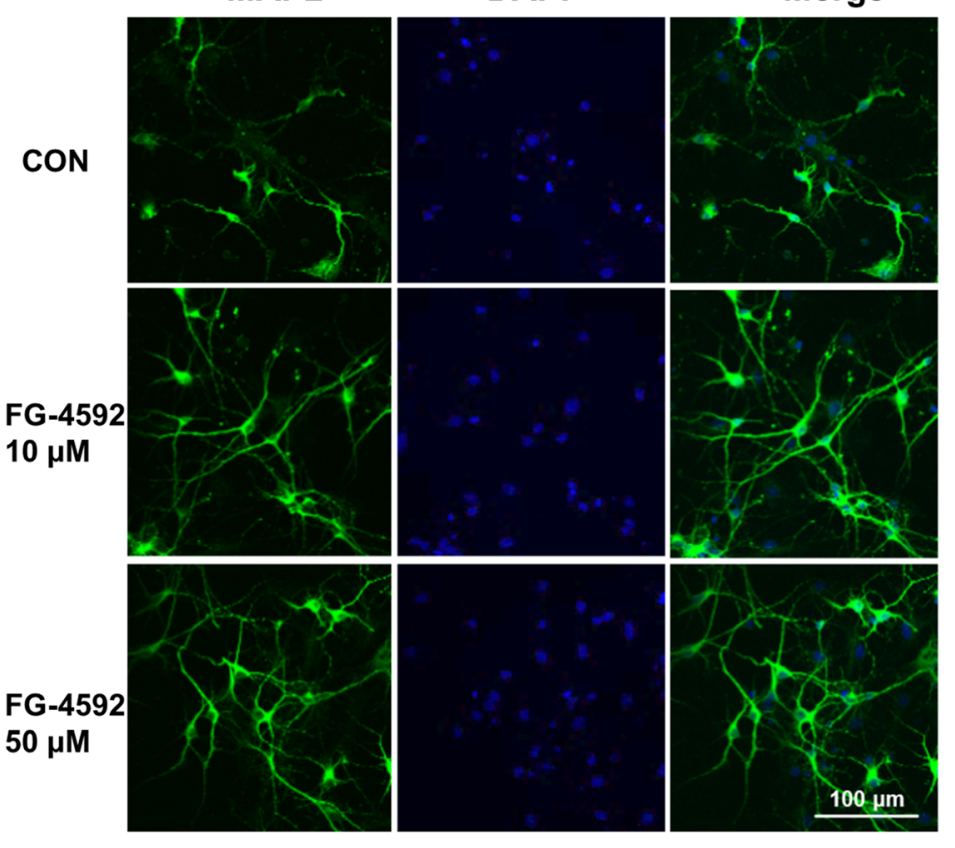

b

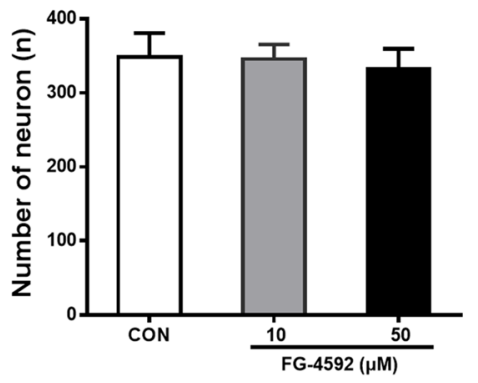

C

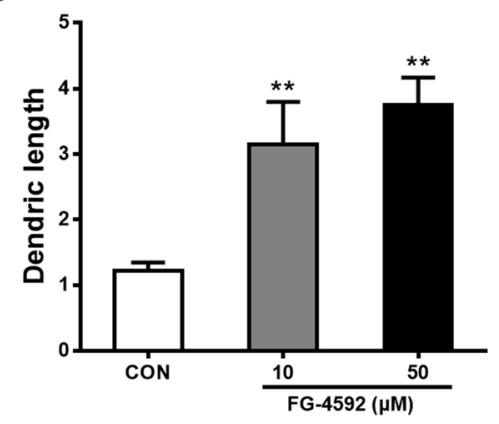

d

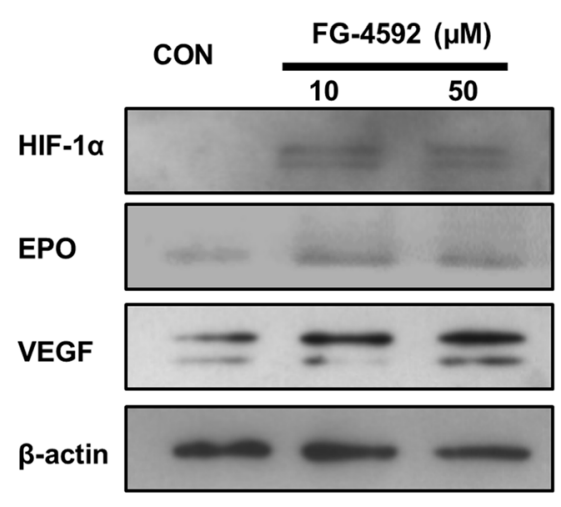

$\mathbf{f}$

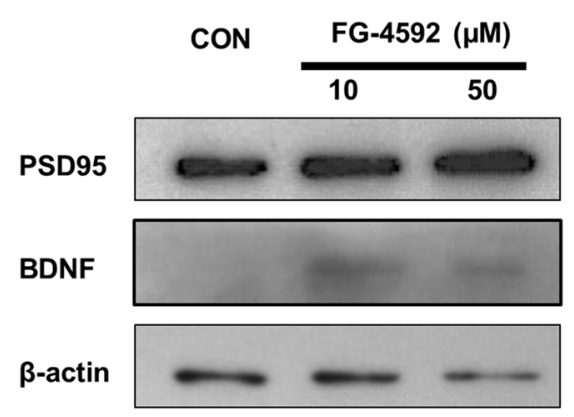

Fig. 6 FG-4592 promoted dendritic growth of primary hippocampal neurons in vitro. (A) Representative confocal images of hippocampal neurons immunostained with anti-microtubule-associated protein 2 (MAP2) antibody reveal dendritic complexity. Scale bar $=50 \mu \mathrm{m}$. (B) Quantification of total neuron numbers. (C) Quantification of total

that FG-4592 has probable synaptic protection benefits. Notably, a report indicated that animals subjected to chronic e

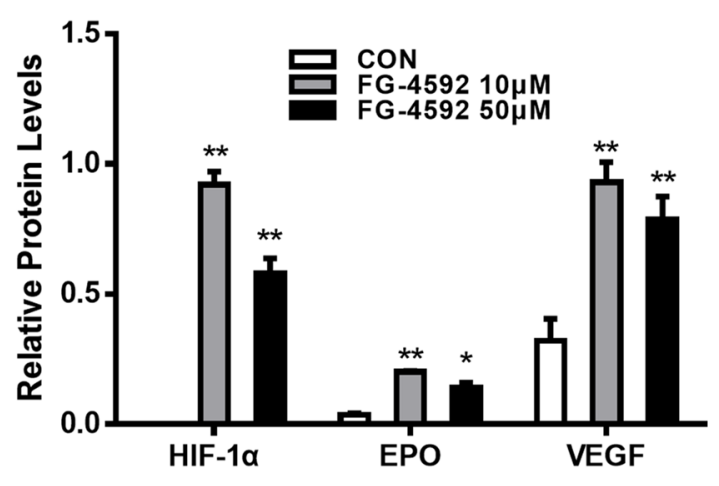

g

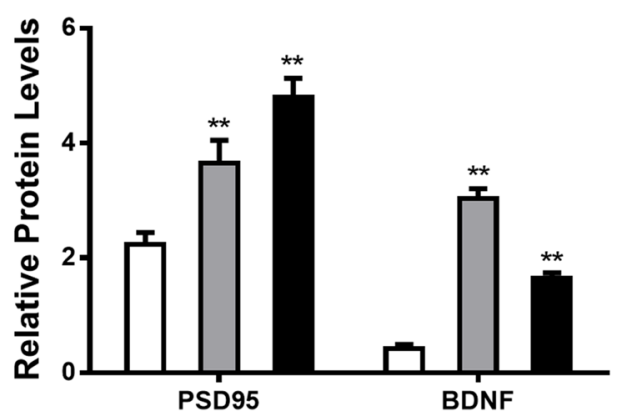

dendritic length. (D-G) Western blot analysis of protein expression in hippocampal neurons treated with or without FG-4592 $(10 \mu \mathrm{M}$ or $50 \mu \mathrm{M})$. Quantification of proteins levels is shown in (E) and (G), respectively. All data are expressed as mean \pm SEM, $n=3,{ }^{*} p<0.05$, $* * p<0.01$ versus control (CON) group

intermittent hypoxia (CIH) showed an improved rate of recovery of synaptic transmission following re-oxygenation in both 


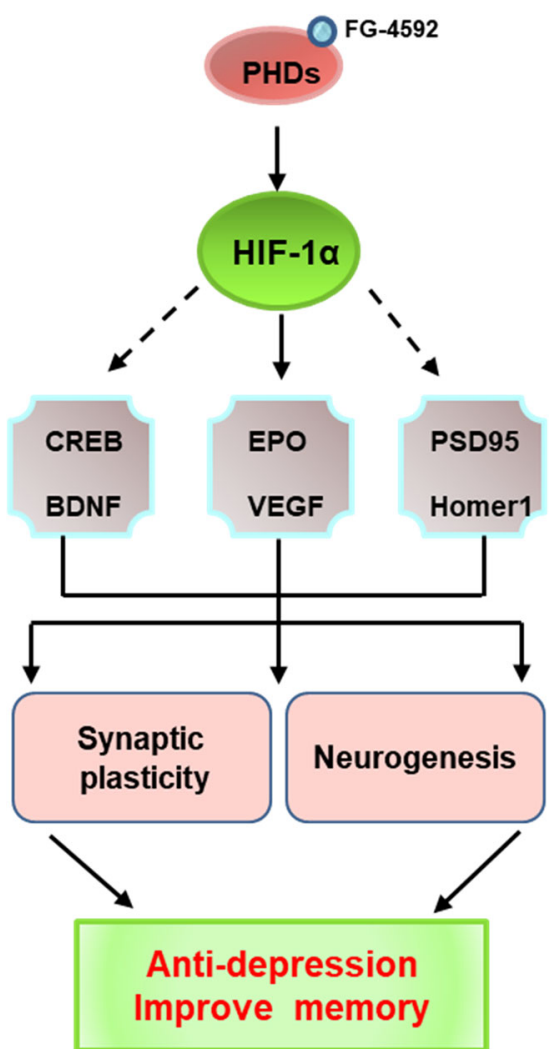

Fig. 7 Schematic model of FG-4592 activity on depression. Schematic representation of molecular mechanisms underlying the protective effect of FG-4592 on depression and memory impairment by activation of hypoxia-inducible factor-1 (HIF-1) signaling after inhibition of prolyl hydroxylase enzyme activity. Brain-derived neurotrophic factor (BDNF) and postsynaptic density protein 95 (PSD95) expression increased when the HIF signaling pathway was activated, eventually leading to increased hippocampal neurogenesis and synaptic plasticity that elicit exerts antidepressant effects

the CA1 and dentate gyrus. However, long-term potential (LTP) induction in the CA1 region and dentate gyrus responded differently to treatment with $\mathrm{CIH}$ or PHD inhibitor DMOG. Therefore, the detailed effect of PHD inhibitors on electrophysiology needs further investigation [35]. When localized in postsynaptic terminals, PSD95 has an important role in postsynaptic function and plasticity [36, 37]. Reportedly, PSD95 binds and stabilizes $N$-methyl-D-aspartate-type glutamate receptors in the post synapse, which is necessary for induction of LTP [38]. Indeed, loss of PSD95 results in severe cognitive decline due to neuronal loss and synaptic disruption [38]. Consistent with a previous report indicating that CUMS caused a reduction in spine density of hippocampal CA1 pyramidal neurons, which was accompanied by a decrease in the level of PSD95 [39], decreased PSD95 expression and impaired spine synaptic plasticity were detected in our CUMS model. These results suggest that FG4592 may enhance synaptic plasticity by promoting BDNF, PSD95, and Homer1 expression in neuronal cells of CUMS model rats. Considering that FG-4592 promoted dendritic growth in primary neurons, it may be involved in dendritic outgrowth by regulating the preservation of synaptic proteins, such as PSD95.

In summary, this study demonstrated that FG-4592 effectively reverses depression-like behavior and memory impairment by virtue of its facilitating role in neurogenesis and synaptic plasticity. The observed beneficial effects are probably attributable to activation of HIF-1 pathway and increased expression of memory-related proteins, such as BDNF, PSD95, and Homer1. Moreover, previous authorization of FG-4592 for clinical application is expected to pave the way for its therapeutic use in the treatment of depression.

Acknowledgments This work was supported by a key grant from Nature and Sciences Foundation of China (No. 81430044) and a grant from Beijing Municipal Science and Technology Commission (No. Z161100000216134).

Required Author Forms Disclosure forms provided by the authors are available with the online version of this article.

Compliance with Ethical Standards All experimental procedures were approved by the Institutional Animal Care and Use Committee of Institute of Cognition and Brain Sciences (No. IACUC2017026) and performed in accordance with the guidelines.

\section{References}

1. Halldorsdottir T, Piechaczek C, Soares de Matos AP, et al. Polygenic risk: predicting depression outcomes in clinical and epidemiological cohorts of youths. Am J Psychiatry. 2019: appiajp201918091014.

2. Surget A, Saxe M, Leman S, et al. Drug-dependent requirement of hippocampal neurogenesis in a model of depression and of antidepressant reversal. Biol Psychiatry. 2008;64:293-301.

3. Porcelli S, Van Der Wee N, van der Werff S, et al. Social brain, social dysfunction and social withdrawal. Neurosci Biobehav Rev. 2019;97:10-33.

4. Slavich GM, Irwin MR. From stress to inflammation and major depressive disorder: a social signal transduction theory of depression. Psychol Bull. 2014;140:774-815.

5. Yirmiya R, Rimmerman N, Reshef R. Depression as a microglial disease. Trends Neurosci. 2015;38:637-58.

6. Chi X, Wang S, Baloch Z, et al. Research progress on classical traditional Chinese medicine formula Lily Bulb and Rehmannia Decoction in the treatment of depression. Biomed Pharmacother. 2019;112:108616.

7. Joshi A. Selective serotonin re-uptake inhibitors: an overview. Psychiatr Danub. 2018;30:605-9.

8. Cipriani A, Zhou X, Del Giovane C, et al. Comparative efficacy and tolerability of antidepressants for major depressive disorder in children and adolescents: a network meta-analysis. Lancet. 2016;388: 881-90.

9. Bouslama M, Adla-Biassette H, Ramanantsoa N, et al. Protective effects of intermittent hypoxia on brain and memory in a mouse model of apnea of prematurity. Front Physiol. 2015;6:313.

10. Zhu XH, Yan HC, Zhang J, et al. Intermittent hypoxia promotes hippocampal neurogenesis and produces antidepressant-like effects in adult rats. J Neurosci. 2010;30:12653-63. 
11. Kushwah N, Jain V, Deep S, et al. Neuroprotective role of intermittent hypobaric hypoxia in unpredictable chronic mild stress induced depression in rats. PLoS One. 2016;11:e0149309.

12. Girgenti MJ, Hunsberger J, Duman CH, et al. Erythropoietin induction by electroconvulsive seizure, gene regulation, and antidepressant-like behavioral effects. Biol Psychiatry. 2009;66:267-74.

13. Deyama S, Bang E, Wohleb ES, et al. Role of neuronal VEGF signaling in the prefrontal cortex in the rapid antidepressant effects of ketamine. Am J Psychiatry. 2019;176:388-400.

14. Huang YT, Liu CH, Yang YC, et al. ROS- and HIF1alphadependent IGFBP3 upregulation blocks IGF1 survival signaling and thereby mediates high-glucose-induced cardiomyocyte apoptosis. J Cell Physiol. 2019;234:13557-70.

15. Gupta N, Wish JB. Hypoxia-inducible factor prolyl hydroxylase inhibitors: a potential new treatment for anemia in patients with CKD. Am J Kidney Dis. 2017;69:815-26.

16. Feng G, Mellor RH, Bernstein M, et al. Imaging neuronal subsets in transgenic mice expressing multiple spectral variants of GFP. Neuron. 2000;28:41-51.

17. Wu K, Zhou K, Wang Y, et al. Stabilization of HIF-1alpha by FG4592 promotes functional recovery and neural protection in experimental spinal cord injury. Brain Res. 2016;1632:19-26.

18. Beck J, Henschel C, Chou J, et al. Evaluation of the carcinogenic potential of roxadustat (FG-4592), a small molecule inhibitor of hypoxia-inducible factor prolyl hydroxylase in CD-1 mice and Sprague-Dawley rats. Int J Toxicol. 2017;36:427-39.

19. Groenendaal-van de Meent D, den Adel M, Rijnders S, et al. The hypoxia-inducible factor prolyl-hydroxylase inhibitor roxadustat (FG-4592) and warfarin in healthy volunteers: a pharmacokinetic and pharmacodynamic drug-drug interaction study. Clin Ther. 2016;38:918-28.

20. Darcet F, Gardier AM, Gaillard R, et al. Cognitive dysfunction in major depressive disorder. a translational review in animal models of the disease. Pharmaceuticals (Basel). 2016;9.

21. Chen Q, Ren L, Min S, et al. Changes in synaptic plasticity are associated with electroconvulsive shock-induced learning and memory impairment in rats with depression-like behavior. Neuropsychiatr Dis Treat. 2018;14:1737-46.

22. Micheli L, Ceccarelli M, D'Andrea G, et al. Depression and adult neurogenesis: positive effects of the antidepressant fluoxetine and of physical exercise. Brain Res Bull. 2018;143:181-93.

23. He M, Wei JX, Mao M, et al. Synaptic plasticity in PTSD and associated comorbidities: the function and mechanism for diagnostics and therapy. Curr Pharm Des. 2018;24:4051-9.

24. Kim YR, Kwon MY, Pak ME, et al. Beneficial effects of GagamPalmultang on scopolamine-induced memory deficits in mice. Evid Based Complement Alternat Med. 2018;2018:3479083.

25. Schega L, Peter B, Torpel A, et al. Effects of intermittent hypoxia on cognitive performance and quality of life in elderly adults: a pilot study. Gerontology. 2013;59:316-23.
26. Deyama S, Bang E, Kato T, Li XY, Duman RS. Neurotrophic and antidepressant actions of brain-derived neurotrophic factor require vascular endothelial growth factor. Biol Psychiatry. 2019;86:143-52.

27. Eriksson PS, Perfilieva E, Bjork-Eriksson T, et al. Neurogenesis in the adult human hippocampus. Nat Med. 1998;4:1313-7.

28. Lucassen PJ, Oomen CA, Naninck EF, et al. Regulation of adult neurogenesis and plasticity by (early) stress, glucocorticoids, and inflammation. Cold Spring Harb Perspect Biol. 2015;7:a021303.

29. Santarelli L, Saxe M, Gross C, et al. Requirement of hippocampal neurogenesis for the behavioral effects of antidepressants. Science. 2003;301:805-9.

30. Thakare VN, Patil RR, Oswal RJ, et al. Therapeutic potential of silymarin in chronic unpredictable mild stress induced depressivelike behavior in mice. J Psychopharmacol. 2018;32:223-35.

31. Jin K, Zhu Y, Sun Y, et al. Vascular endothelial growth factor (VEGF) stimulates neurogenesis in vitro and in vivo. Proc Natl Acad Sci U S A. 2002;99:11946-50.

32. Sun $\mathrm{C}, \mathrm{Fu} \mathrm{J}, \mathrm{Qu} \mathrm{Z}$, et al. Chronic mild hypoxia promotes hippocampal neurogenesis involving Notch1 signaling in epileptic rats. Brain Res. 2019;1714:88-98.

33. Benedetti F, Poletti S, Locatelli C, et al. A Homer 1 gene variant influences brain structure and function, lithium effects on white matter, and antidepressant response in bipolar disorder: a multimodal genetic imaging study. Prog Neuropsychopharmacol Biol Psychiatry. 2018;81:88-95.

34. Shiraishi Y, Mizutani A, Mikoshiba K, et al. Coincidence in dendritic clustering and synaptic targeting of homer proteins and NMDA receptor complex proteins NR2B and PSD95 during development of cultured hippocampal neurons. Mol Cell Neurosci. 2003;22:188-201.

35. Wall AM, Corcoran AE, O'Halloran KD, O'Connor JJ. Effects of prolyl-hydroxylase inhibition and chronic intermittent hypoxia on synaptic transmission and plasticity in the rat CA1 and dentate gyrus. Neurobiol Dis. 2014;62:8-17.

36. El-Husseini AE, Schnell E, Chetkovich DM, et al. PSD-95 involvement in maturation of excitatory synapses. Science. 2000;290: 1364-8.

37. Kim MJ, Futai K, Jo J, et al. Synaptic accumulation of PSD-95 and synaptic function regulated by phosphorylation of serine-295 of PSD-95. Neuron. 2007;56:488-502.

38. Han K, Kim E. Synaptic adhesion molecules and PSD-95. Prog Neurobiol. 2008;84:263-83.

39. Qiao H, An SC, Xu C, et al. Role of proBDNF and BDNF in dendritic spine plasticity and depressive-like behaviors induced by an animal model of depression. Brain Res. 2017;1663:29-37.

Publisher's Note Springer Nature remains neutral with regard to jurisdictional claims in published maps and institutional affiliations. 